\title{
The EAP Treatment for the School Children's Diseases
}

\section{(1) The Improvement of the Colour-Sense of the Colour-Blind Children \\ (2) The Improvement of the Vision-Power of Pseudomyopia}

\author{
Dr. Y. Narimiya (Tokyo) \\ Dr. Y. Nozu (Tokyo) \\ Dr. Y. Nakatani (Osaka) \\ Dr. Y. Tsuyama (Kanazawa) \\ Dr. M. Nakamura (Tokyo) \\ and 34 Doctors
}

\section{Introduction}

24 years ago at Kyoto University while studying the resistance of electric current in skin, Dr. Y. Nakatani discovered by his Neurometer Ryodo Points, Ryodoraku and Hanno-Ryodo Points (Reactive Electro Permeable Points); and applying stimulation with an electric needles at his Hanno Ryodo Points, he has had good results in treating newralgia, colour blindness, psendramyopia, and many other chrinic diseases. This is called Ryodoraku Autonomous Nerve Regulatory Therapy. In 1563 at Rome, in 1967 at Prague, and in 1971 at Lisbon Dr. Y. Nozu made a report regarding the improvement of the colour sense of the Ryodoraku Treatment at the International Congress of School and University Health and Medicine.

Last Year (1973) Prof. N. Sato made a report of Ryodoraku Therapy at the International Symposium On Pain at Seattle, Washington, U.S.A.

\section{Method}

We treat with the doctrine of Ryodoraku Therapy, which acupunctures at Nakatanis 4 points on the face and foot, and turn on a direct current of $12 \mathrm{~V} \& 200$ M.A. for 7 seconds. With this method, the colour vision are getting better.

(Table I)

\begin{tabular}{|c|c|c|c|c|c|c|}
\hline $\begin{array}{c}\text { Ishiwara } \\
10 \\
\text { Tables }\end{array}$ & $\begin{array}{l}\text { The num- } \\
\text { ber of total } \\
\text { pupils }\end{array}$ & $\begin{array}{l}\text { The num- } \\
\text { ber of the } \\
\text { colour } \\
\text { blindness }\end{array}$ & $\begin{array}{l}\text { The num. } \\
\text { ber of } \\
\text { correct } \\
\text { reading }\end{array}$ & $\begin{array}{l}\text { After 15th } \\
\text { treatment }\end{array}$ & $\begin{array}{l}\text { Increased } \\
\text { number of } \\
\text { correct } \\
\text { reading }\end{array}$ & $\begin{array}{l}\text { Average } \\
\text { treatment } \\
\text { requried for } \\
\text { correct read- } \\
\text { ing of each } \\
\text { Table }\end{array}$ \\
\hline $\begin{array}{l}\text { Elementary } \\
\text { School } \\
\text { Pupils }\end{array}$ & $\begin{array}{lr}\text { 1. } & 693 \\
\text { 2. } & 1291 \\
\text { 3. } & 1063\end{array}$ & $\begin{array}{l}14 \\
11 \\
13\end{array}$ & $\begin{array}{l}63 \\
20 \\
35\end{array}$ & $\begin{array}{r}100 \\
54 \\
97\end{array}$ & $\begin{array}{l}37 \\
34 \\
62\end{array}$ & $\begin{array}{l}5.68 \\
4.85 \\
3.15\end{array}$ \\
\hline $\begin{array}{l}\text { High School } \\
\text { Pupils } \\
\text { Total }\end{array}$ & $\begin{array}{rr}\text { 4. } & 460 \\
5 . & 752 \\
& 4259\end{array}$ & $\begin{array}{l}14 \\
10 \\
62\end{array}$ & $\begin{array}{r}30 \\
26 \\
174\end{array}$ & $\begin{array}{r}89 \\
59 \\
399\end{array}$ & $\begin{array}{r}59 \\
33 \\
225\end{array}$ & $\begin{array}{l}3.56 \\
4.54 \\
\\
4.13\end{array}$ \\
\hline
\end{tabular}




\section{The Results}

In the next tables, it is quite clear that the colour sense of the colour blind school children has improved.

(1) The topics applied for the school children as group.

(2) Dr. Y. Tuyama's investigation for 145 middle school pupils.

(Table II)

\begin{tabular}{|l|c|c|c|c|}
\hline \multicolumn{1}{|c|}{ Result } & $\begin{array}{c}\text { Ishiwara's } \\
10 \text { Tables }\end{array}$ & 1968 & 1969 & Total \\
\hline Improved & $10 / 10$ & $\begin{array}{c}34 \\
(68 \%)\end{array}$ & $\begin{array}{c}65 \\
(69 \%)\end{array}$ & $\begin{array}{c}99 \\
(68 \%)\end{array}$ \\
\hline $\begin{array}{l}\text { Half } \\
\text { improved }\end{array}$ & $5 / 10$ & $\begin{array}{c}9 \\
(18 \%)\end{array}$ & $\begin{array}{c}28 \\
(30 \%)\end{array}$ & $\begin{array}{c}37 \\
(25 \%)\end{array}$ \\
\hline Favourable & $1 \sim 4 / 10$ & $\begin{array}{c}7 \\
(14 \%)\end{array}$ & $\begin{array}{c}2 \\
(2 \%)\end{array}$ & $\begin{array}{c}9 \\
(7 \%)\end{array}$ \\
\hline Total & & 50 cases & 95 cases & 145 cases \\
\hline
\end{tabular}

(3) Dr. Y. Narimiya's investigation for elementary, middle, and high school pupils, college students and adults.

(Table III)

\begin{tabular}{|c|c|c|c|c|c|c|c|}
\hline $\begin{array}{l}\text { School } \\
\text { groups }\end{array}$ & $\begin{array}{l}\text { Ishiwara } \\
\text { Tables }\end{array}$ & $\begin{array}{l}\text { No. of } \\
\text { colour } \\
\text { blindness }\end{array}$ & $\begin{array}{l}\text { No. of } \\
\text { correct } \\
\text { reading }\end{array}$ & $\begin{array}{l}\text { After } \\
\text { 15th } \\
\text { treatment }\end{array}$ & $\begin{array}{l}\text { All No. } \\
\text { of correct } \\
\text { reading }\end{array}$ & $\begin{array}{l}\text { No. of } \\
\text { total } \\
\text { treatment }\end{array}$ & $\begin{array}{l}\text { Average } \\
\text { treatment } \\
\text { required for } \\
\text { reading of } \\
\text { each Table }\end{array}$ \\
\hline $\begin{array}{l}\text { Elementary } \\
\text { school } \\
\text { pupils }\end{array}$ & $\begin{array}{l}10 \\
12 \\
14\end{array}$ & $\begin{array}{r}11 \\
4 \\
6\end{array}$ & $\begin{array}{l}20 \\
24 \\
19\end{array}$ & $\begin{array}{l}79 \\
42 \\
48\end{array}$ & $\begin{array}{l}83 \\
60 \\
81\end{array}$ & $\begin{array}{r}175 \\
61 \\
101\end{array}$ & $\begin{array}{l}10.2 \\
16 \\
10.5\end{array}$ \\
\hline $\begin{array}{l}\text { Middle } \\
\text { school } \\
\text { pupils }\end{array}$ & $\begin{array}{l}10 \\
12 \\
14\end{array}$ & $\begin{array}{r}11 \\
8 \\
6\end{array}$ & $\begin{array}{l}34 \\
28 \\
24\end{array}$ & $\begin{array}{l}74 \\
72 \\
56\end{array}$ & $\begin{array}{l}158 \\
141 \\
174\end{array}$ & $\begin{array}{r}94 \\
87 \\
105\end{array}$ & $\begin{array}{r}11 \\
8.9 \\
10.3\end{array}$ \\
\hline $\begin{array}{l}\text { High } \\
\text { school } \\
\text { pupils }\end{array}$ & $\begin{array}{l}10 \\
12 \\
14\end{array}$ & $\begin{array}{l}8 \\
8 \\
8\end{array}$ & $\begin{array}{l}24 \\
28 \\
32\end{array}$ & $\begin{array}{l}57 \\
51 \\
53\end{array}$ & $\begin{array}{l}64 \\
57 \\
67\end{array}$ & $\begin{array}{l}209 \\
196 \\
212\end{array}$ & $\begin{array}{c}6.3 \\
10.2 \\
6\end{array}$ \\
\hline $\begin{array}{l}\text { College } \\
\text { students }\end{array}$ & $\begin{array}{l}10 \\
12 \\
14\end{array}$ & $\begin{array}{l}7 \\
4 \\
5\end{array}$ & $\begin{array}{l}23 \\
11 \\
19\end{array}$ & $\begin{array}{l}61 \\
42 \\
41\end{array}$ & $\begin{array}{l}79 \\
54 \\
63\end{array}$ & $\begin{array}{l}148 \\
102 \\
128\end{array}$ & $\begin{array}{l}7.8 \\
9.9 \\
9.8\end{array}$ \\
\hline Adults & $\begin{array}{l}10 \\
12 \\
14\end{array}$ & $\begin{array}{l}9 \\
6 \\
9\end{array}$ & $\begin{array}{l}17 \\
13 \\
18\end{array}$ & $\begin{array}{l}51 \\
27 \\
58\end{array}$ & $\begin{array}{r}91 \\
48 \\
141\end{array}$ & $\begin{array}{l}232 \\
269 \\
325\end{array}$ & $\begin{array}{l}12.1 \\
16.5 \\
15.4\end{array}$ \\
\hline Total & $\begin{array}{l}10 \\
12 \\
14\end{array}$ & $\begin{array}{l}45 \\
30 \\
34\end{array}$ & $\begin{array}{l}118 \\
104 \\
112\end{array}$ & $\begin{array}{l}433 \\
346 \\
256\end{array}$ & $\begin{array}{l}475 \\
360 \\
526\end{array}$ & $\begin{array}{l}858 \\
715 \\
871\end{array}$ & $\begin{array}{r}9.8 \\
11.8 \\
10.6\end{array}$ \\
\hline
\end{tabular}


(4) Total of Dr. Y. Nakatani and other 21 doctor's investigation for 1039.

(Table IV)

\begin{tabular}{|l|c|c|c|c|}
\hline $\begin{array}{l}\text { Ishiwara } \\
\text { Tables }\end{array}$ & $\begin{array}{l}\text { Number of } \\
\text { colour } \\
\text { blindness }\end{array}$ & $\begin{array}{l}\text { Number of } \\
\text { treatment }\end{array}$ & $\begin{array}{l}\text { Number of } \\
\text { correct } \\
\text { reading }\end{array}$ & $\begin{array}{l}\text { Average treatment } \\
\text { required for } \\
\text { correct reading } \\
\text { of each Table }\end{array}$ \\
\hline 10 Tables & 1039 & 16939 & 5396 & 3.13 \\
12 Tables & 304 & 4355 & 1645 & 2.64 \\
14 Tables & 552 & 9628 & 3705 & 2.59 \\
\hline
\end{tabular}

III' Follow-up Tests by Ishiwara Colour-blind Tables.

It is clear that the colour sense of the school children will be remained improved after 1,2 and 3 years.

(1) Dr. Y. Tsuyama's investigation for 21 middle school pupils (Ishiwara 10 Tables)

(Table V)

\begin{tabular}{|c|c|c|c|c|c|c|c|c|c|}
\hline & & \multicolumn{7}{|c|}{ Percentage of correct reading } \\
\cline { 2 - 9 } & $100 \%$ & 90 & 80 & 50 & 40 & 30 & 20 & 10 & Total \\
\hline 1 year & 1 & & 1 & 1 & 1 & 6 & & 1 & 11 \\
3 years & 2 & & & & & & & & 2 \\
6 years & 3 & 1 & & 1 & & 2 & 1 & & 8 \\
\hline
\end{tabular}

(2) Dr. Y. Narimiya's investigation for elementary, middle \& high school pupils, and college students. (total 8)

(Ishiwara 10, 12, 14 Tables)

c. f. one of them, a middle school pupils. (Table VI)

(Table VI)

\begin{tabular}{|c|c|c|c|c|c|c|c|c|c|}
\hline \multirow[b]{2}{*}{ After } & \multicolumn{3}{|c|}{10 Tables } & \multicolumn{3}{|c|}{12 Tables } & \multicolumn{3}{|c|}{14 Tables } \\
\hline & $\begin{array}{l}\text { No. of } \\
\text { correct } \\
\text { reading } \\
\text { before } \\
\text { treat- } \\
\text { ment }\end{array}$ & $\begin{array}{l}\text { No. of } \\
\text { treat- } \\
\text { ment }\end{array}$ & $\begin{array}{l}\text { No. of } \\
\text { correct } \\
\text { reading } \\
\text { after } \\
\text { treat- } \\
\text { ment }\end{array}$ & $\begin{array}{l}\text { No. of } \\
\text { correct } \\
\text { reading } \\
\text { before } \\
\text { treat- } \\
\text { ment }\end{array}$ & $\begin{array}{l}\text { No. of } \\
\text { treat- } \\
\text { ment }\end{array}$ & $\begin{array}{l}\text { No. of } \\
\text { correct } \\
\text { reading } \\
\text { after } \\
\text { treat- } \\
\text { ment }\end{array}$ & $\begin{array}{l}\text { No. of } \\
\text { correct } \\
\text { reading } \\
\text { before } \\
\text { treat- } \\
\text { ment }\end{array}$ & $\begin{array}{l}\text { No. of } \\
\text { treat- } \\
\text { ment }\end{array}$ & $\begin{array}{l}\text { No. of } \\
\text { correct } \\
\text { reading } \\
\text { after } \\
\text { treat- } \\
\text { ment }\end{array}$ \\
\hline & 3 & 8 & 9 & 5 & 8 & 8 & 4 & 8 & 10 \\
\hline 1 year & 8 & 3 & $\underline{10}$ & 7 & 7 & $\underline{12}$ & 8 & 7 & 13 \\
\hline 3 years & 8 & 3 & 10 & 11 & 3 & 12 & 10 & 3 & 13 \\
\hline 6 years & $\underline{10}$ & 4 & 10 & 12 & 5 & 12 & 13 & 4 & 14 \\
\hline
\end{tabular}

\section{Pseudomyopia}

In the next graph (12 doctor's, study for 280 pupils), it is quite clear that the vision power of pseudomyopia will be improved by Ryodoraku Treatment (Table VII). 
(Table VII)

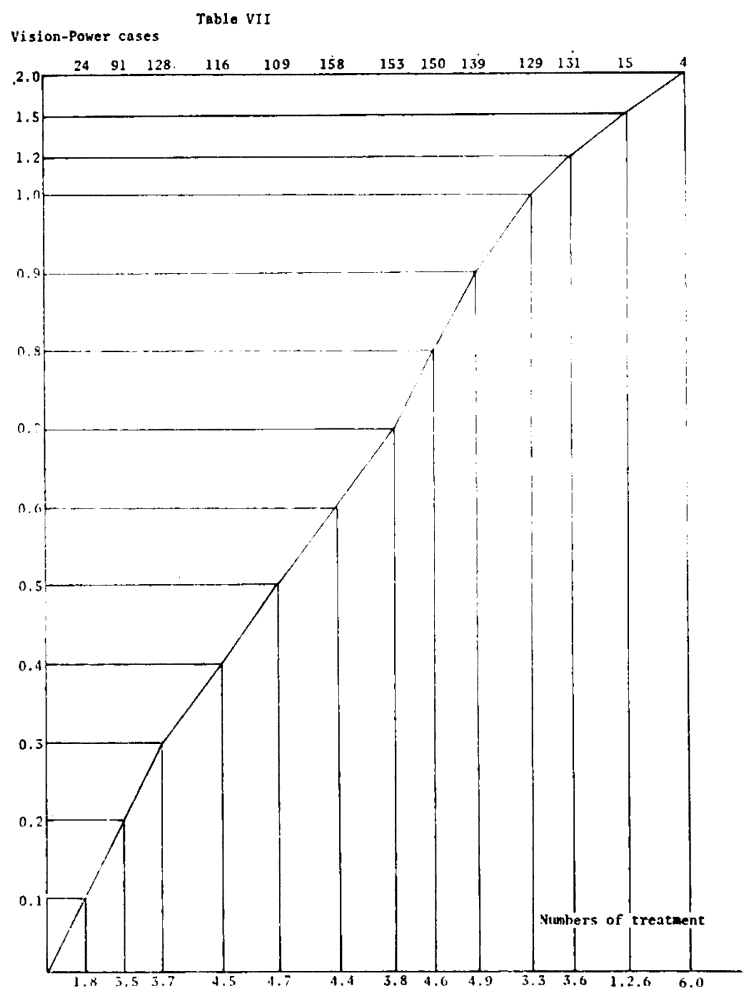

v. Summary

This time, we report the results of the group treatment of the colour blind and pseudomyopia. Perhaps we can say that the improvement of colour sense and pseudomyopia is possible by Ryodoraku Treatment. As the result, many colour blind school children can pass the entrance examination of a medical college, and also the examination to be automobile drivers. Although, they can read the Ishiwara Colour Blind Tables, we cannot say that they are completely cured.

Prof. R. Seki, an ophthalmologist examined some of them (14 cases) by anmalos cope, Ishikawa's lantern test, Ishiwara's Tables, Okuma's Tables, TOKYO Medical College Tables, 100 hue test and Panel D-15 test; and the improvement of the colour sense is observed, but the result is not yet perfect afterwards, we show you some demonstration, and I hope, all of you will try this method. 